resses differ enormously in total fecundity yet, rather than producing the same proportion of males, each makes approximately the same absolute number. We are not convinced that Yamaguchi's simple and original theoretical explanation for this pattern captures all of the relevant features of the biology of the aphids, as it seems to assume that a female can assess the total fecundity of the foundress group to which she belongs; this would be reasonable for fig wasps, which produce their offspring after arriving at the fig, but may not be reasonable for the aphids studied by Yamaguchi, which produce their offspring before they fly to the trees on which the foundress groups are formed. But the pattern is there. It invites more theorizing and, of course, more empirical work.

$\mathrm{XX}-\mathrm{XO}$ systems occur in many groups of arthropods and seem often to be associated with complex sex-allocation strategies (as in the laboratory nematode
Caenorhabditis elegans, which is mainly hermaphroditic, but with a small proportion of males). Yamaguchi's data should stimulate a widened search for instances of extreme sex-ratio manipulation among $\mathrm{XX}-\mathrm{XO}$ species. Aphids themselves are economically important, so it may prove relatively easy to obtain support for studying their sex lives.

1. Darwin. C. The Descent of Matn. and Salection in Relation wo Sex (Murray. London. 1871 : reprinted by Princeton University Press. $\mid 9811$

Herre, E.A. Science 228. 846 (1985)

3. Yamaguchi. Y. Nature $318,+6)(1985)$

4. Fisher. R.A. The Genetical Theory of Natural Solection (Clarendon. Oxford. 1931): reprinted hy Dover. New York.

(Clare
1958 ).

5. Hamilton. W.D. Science 156. 477 (1967)

6. Bult. J.J. Evolution of $S_{C x}$ Detcrmining Mechanisms (Benjamin/Cummings. Menlo Pasrk. 1983).

. Charnov. E.L. The Theory of Sex Allecation (Princeton University Press. 1982)

8. Trivers. R. Social Evolution (Benjamin/Cummings, Menlo Park. 1985).

Robert M. May and Jon Seger are in the Department of Biology at Princeton University, Princeton, New Jersey 08544, USA.

\title{
Quantum Hall effect
}

\section{Dimensionality leaves its mark}

\section{from R.J. Haug}

WhAT happens when one plays with the dimensionality of an object? This is not an esoteric question of interest only to mathematicans but a very important problem that has been actively explored by solid-state physicists in the past few years, not least because it is likely to lead to many totally new physical phenomena. Already, measurements of a two-dimensional electron gas (2DEG) in strong magnetic fields have revealed the quantum Hall effect (von Klitzing, K., Dorda, G. \& Pepper, M. Phys. Rev. Lett. 45, 494; 1980), for which von Klitzing was awarded this year's Nobel Prize in Physics. Now, H.Z. Zheng, K.K. Choi, D.C. Tsui and G. Weimann (Phys. Rev. Lett. 55, 1144; 1985) have found a new, but related, effect - an anomalous variation in the magneto-transport coefficients of a twodimensional electron gas as the width of the conducting channel becomes smaller than a characteristic length. This is of the order of $10 \mu \mathrm{m}$ depending on the mobility of the electrons. The authors assume that the effect, which was only observed in high-quality samples, arises from a transition from two-dimensional to onedimensional behaviour.

One of the reasons for this branch of research is the need for ever smaller components in the electronics industry. But, in addition, the modulation-doped GaAs/ AlGaAs heterostructures used by Zheng et al. can also be used to fabricate very fast switches (Störmer, H.L., Nature News and Views 317, 20; 1985). One obtains these structures by growing thin layers of GaAs, AlGaAs and AlGaAs doped with silicon on a crystal of GaAs by molecular beam epitaxy (MBE). Because of the different electronic properties of the two semiconducting materials GaAs and AlGaAs, the electrons from the Si donors in the $\mathrm{AlGaAs}$ transfer to the GaAs layer, so forming a quasi-two-dimensional electron gas bound to the interface via the Coulomb attraction of the ionized donors across the junction.

An electron localized in a region of sufficiently small thickness behaves differently from a free electron that is able to move over large distances in all directions. If the electron is confined within a narrow potential well in the third direction, the quantized energy levels become discrete rather than being quasicontinuous. The electrons are unable to move freely in this direction. At low enough temperatures and sufficiently small carrier concentrations all electrons are in the lowest energy level; this is a description of a $2 \mathrm{DEG}$. Owing to the spatial separation of electrons from their ionized parent impurities, the scattering rate is strongly reduced, which leads to exceedingly high mobilities, particularly in the low-temperature regime.

If a strong magnetic field is applied in the $z$ direction perpendicular to a $2 \mathrm{DEG}$ kept at the temperature of liquid helium, and the voltages in the direction of the applied current $\left(V_{\mathrm{x}}\right)$ and perpendicular to this direction and to the magnetic field $\left(V_{\mathrm{y}}\right)$ are measured, a non-vanishing $V$, is found in the magnetic field. This is the effect found in 1879 by E.H. Hall at Johns Hopkins University and it is true for all conducting materials. Von Klitzing observed that in a $2 D E G$ at certain regions $V$ vanishes, although there is still a current $I$ through the sample and the Hall voltage $V$, shows a plateau. In such regions the relation of $V$, to the current $l$ is given by $V_{1} / I=h / \eta e^{2}$, where $h$ is the Planck constant, $e$ the charge of an electron and $\eta$ an integral number.

Zheng et al., like von Klitzing, measured $V$, and $V$, as a function of the magnetic field at low temperatures, with the aim of investigating size effects in the sample by changing the width of the conducting channel. First. they noticed that the quantum Hall effect is exact. When $V$, vanished, the Hall voltage always showed a plateau with $V, / I=h / \eta e^{2}$. This is another proof that the quantum Hall effect is a universal property of twodimensionality of the charge carriers and does not depend on properties of the samples, such as their size.

But Zheng et al. also looked at the lineshape of their experimental curves in the region between the plateaus of the quantum Hall effect. When the electrons had low mobilities no difference was observed between wide and narrow samples. But for samples with very high electronic mobilities, the lineshape changed dramatically when the width of the channel was reduced, although the measurements with a wide conducting channel were still comparable to those obtained with low electronic mobility samples.

The data suggest that the new phenomenon arises as the size of the sample approaches some characteristic length of the electronic system. One possible explanation put forward by Zheng et al. is that the characteristic length is that of the inhomogeneities in the sample. Another possiblity is that it is associated with the electron-electron interaction, in which case the observed effect would have to be attributed to a dimensional crossover from two-dimensional to one-dimensional behaviour. In other words, if the channel is narrow enough the electrons cannot move freely in two dimensions: one direction is open but in the other motion is restricted by the edges of the sample.

These explanations are, however, not definitive. In experiments with silicon metal-oxide semiconductor field-effect transistors (Si-MOSFET), where samples of different channel length but identical channel width have been measured (von Klitzing, K. et al. Proc. 17th inst. Conf. Physics Semiconductors, San Francisco, 1984), very long samples produce results that are strikingly similar to those of Zheng et al. for very narrow GaAs/ AlGaAs samples, whereas short samples do not. For a long sample it is difficult to explain the effect by a dimensional crossover as motion is not restricted in one direction. Nevertheless, the experiments give hints about the underlying fundamental physics. Further experiments and ideas will clarify these problems.

R.J. Haug is at the Max-Planck-Institut für Festkörperforschung. Heisenbergstrasse 1, D-7000 Stuttgart $80, F R G$. 